\title{
Modulation and Demodulation of QPSK Communication System Based on Matlab
}

\author{
Xiaoling $\mathrm{Hu}$ \\ The Engineering \& Technical College of Chengdu University of Technology, \\ Leshan, Sichuan, China, 999078 \\ E-mail: $\underline{80354029 @ q q . c o m}$
}

\begin{abstract}
Currently, multiple modulation schemes based on QPS K are applied more and more widely. They are involved in digital microwa ve communication system, digital satellite communication system, broadband access, mobile communication and cable television system. QPSK is quarternary phase-shift keying. It characterizes digital information input through differences of four different phases of carrier. It specifies four phases of carrier. One dual-bit code element is corresponding to two binary information bits. They can be regarded as the synthesis of two 2PS K signals. In QPS K, each modulation can transmit two information bits. They are transferred through four phases of carrier. This design realizes modulation and demodulation of QPS K on Matlab platform. It then verifies relevant theoretical knowledge of QPS K systematically.
\end{abstract}

Keywords- QPSK ; Matlab; Modulation; Dual-bit Code

\section{INTRODUCTION}

Currently, quarternary phase-shift keying is widely used in digital satellite modulation. Its advantages include strong anti-interference performance, high frequency utilization ratio and easiness to achieve. Common satellite dishes and cable television system use QPSK signal. Currently Uplink or downlink modulation of $3 \mathrm{G}$ and $4 \mathrm{G}$ standards applicable in China mostly uses QPSK. For example, channel noise threshold is as low as $4.5 \mathrm{~dB}$ and transmission code rate reaches $45 \mathrm{Mb} / \mathrm{s}$ in digital satellite television DVB2S standard. The use of QPSK modulation guarantees both signal transmission efficiency and bit error rate performance. This paper clearly shows QPSK modulation and demodulation process on the software. It verifies QPSK core technology systematically.

\section{QPSK MODULATION PRINCIPLE}

Compared to BPSK frequency band, QPSK frequency band has higher efficiency when a single modulation symbol is sending 2 bit. The phase of carrier is only one selected among phases $0, \pi / 2, \pi$ and $3 \pi / 2$. Single space phase refers to the only one pair of bits. Therefore, QPSK signal can be written as ${ }^{[6]}$ :

“Ts” refers to symbol interval:

$$
\begin{aligned}
s_{Q P S K}(t) & =\sqrt{\frac{2 E_{s}}{T_{s}}}\left\{\cos \left(2 \pi f_{c} t\right) \cos \left[(i-1) \frac{\pi}{2}\right]\right. \\
\left.-\sin \left(2 \pi f_{c} t\right) \sin \left[(i-1) \frac{\pi}{2}\right]\right\} & (1) \\
\Phi_{1}(t) & =\sqrt{\frac{2}{T_{s}}} \cos \left(2 \pi f_{c} t\right) \\
\Phi_{2}(t) & =\sqrt{\frac{2}{T_{s}}} \sin \left(2 \pi f_{c} t\right)
\end{aligned}
$$

Then,

$$
\begin{aligned}
& s_{Q P S K}(t)=\sqrt{E_{s}} \cos \left[(i-1) \frac{\pi}{2}\right] \Phi_{1}(t) \\
& -\sqrt{E_{s}} \sin \left[(i-1) \frac{\pi}{2}\right] \Phi_{2}(t)
\end{aligned}
$$

$\mathrm{i}=1,2,3,4$

QPSK generally selects four carriers with different phases to identify digital information. Information in the first place constituting dual-bit code element is identified with a. Information at the tail end is identified with $b$. Dual-bit information is generally arranged according to Gray code sequence.

\section{SIMULATION DESIGN OF QPSK SYSTEM}

The type of carrier used by I signal is cosine which is mainly composed of odd number sequence of binary data stream $^{[12]}$. The type of carrier used by Q signal is sine which is mainly composed of even number sequence of binary data stream. In the figure below, a represents I data and $b$ represents $Q$ data. They output $Q$ signal and $Q$ signal according to their different carrier characteristics. I signal and Q signal transform into QPSK output signal after addition. When I carrier signal is in phase 0 , its value is displayed as 1 ; when it is in phase $180^{\circ}$, its value is displayed as 0 . When $\mathrm{Q}$ carrier signal is in phase 0 , its value is displayed as 1 ; when it is in phase $180^{\circ}$, its value is displayed as 0. According to Fig. 1, a and b signals will transform into QPSK signal after combination. They are identified through red and blue lines. OPSK signal is subject to fast Fourier transform. Its main function 
expression uses fft(QPSK_rc) and its spectrogram is obtained. This spectrogram as shown in Fig. 2 is produced under noise-free conditions. Central frequency 0 point is used as reference. Side frequency is $50 \mathrm{HZ}$.

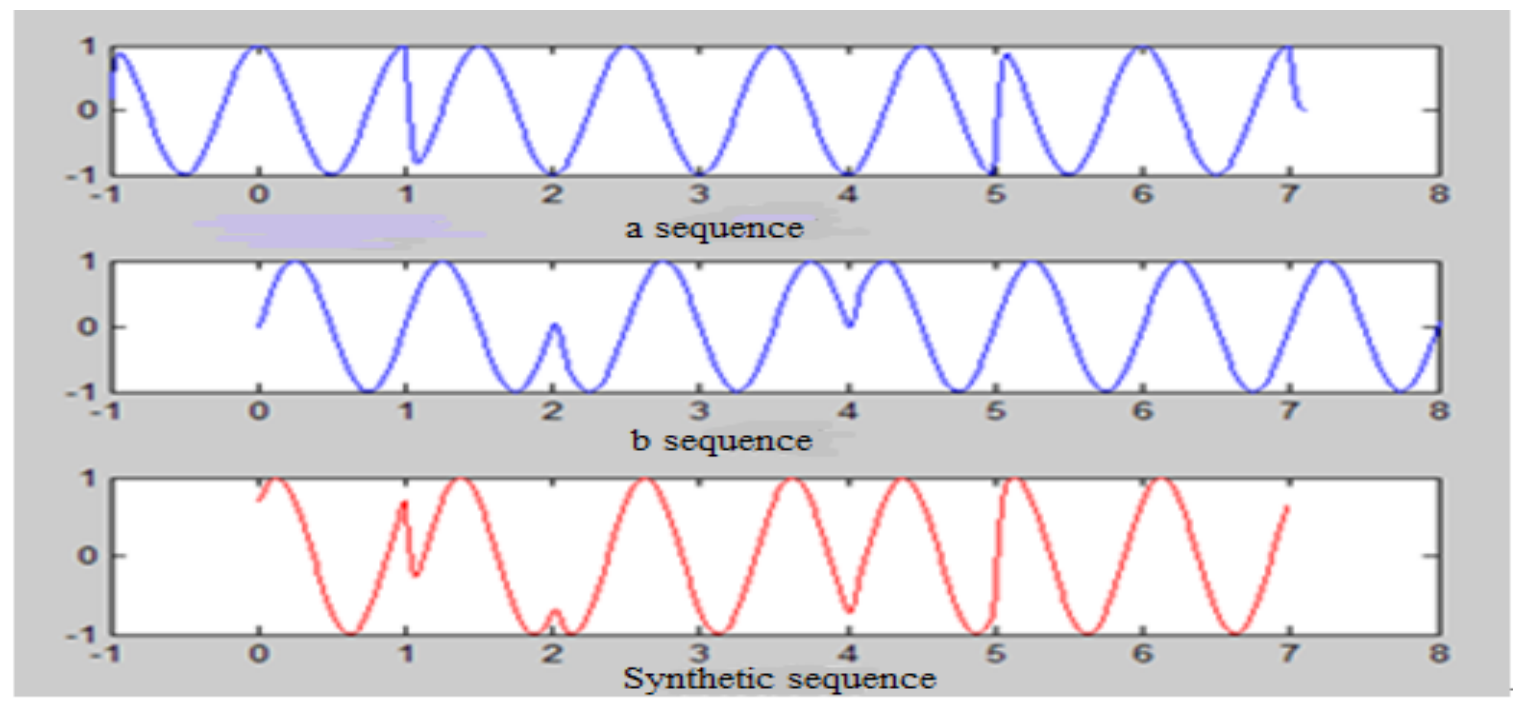

Figure 1. QPSK modulation signal

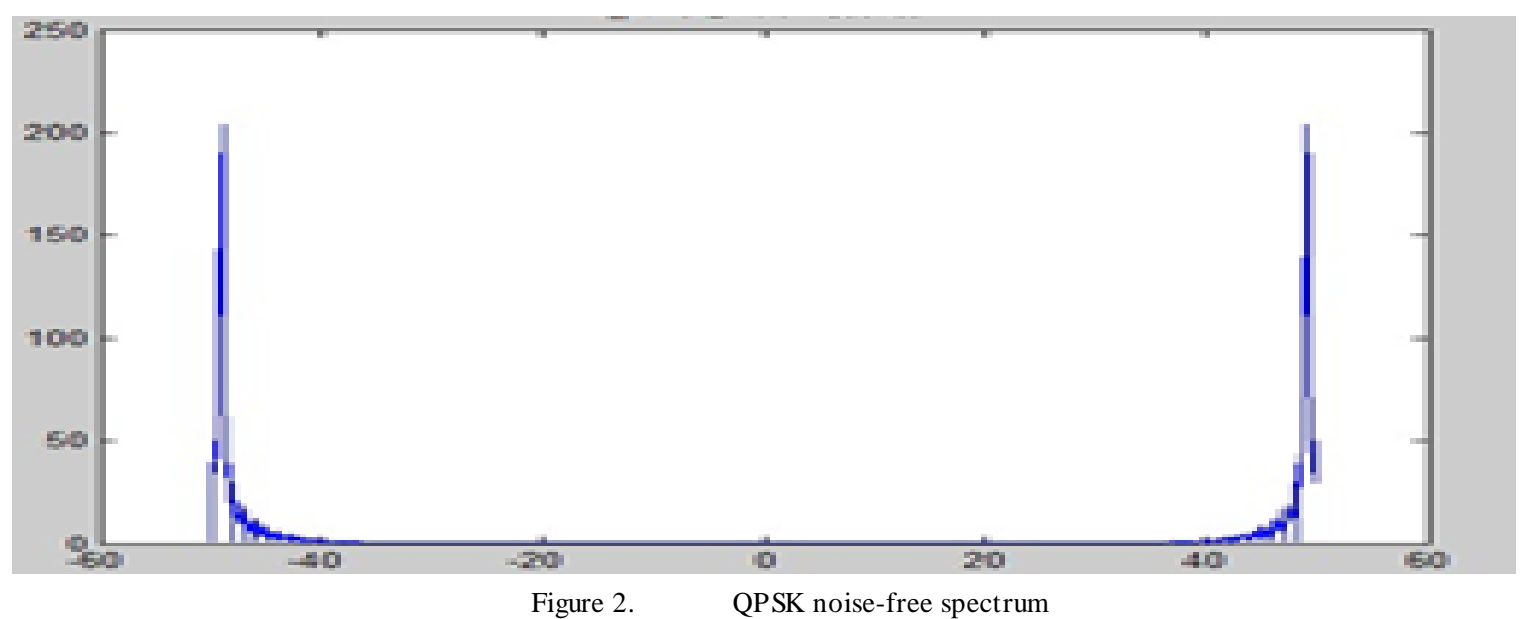

Signal diagram Fig. 3 and spectrogram Fig. 5 of QPSK modulated signal after the addition of noise

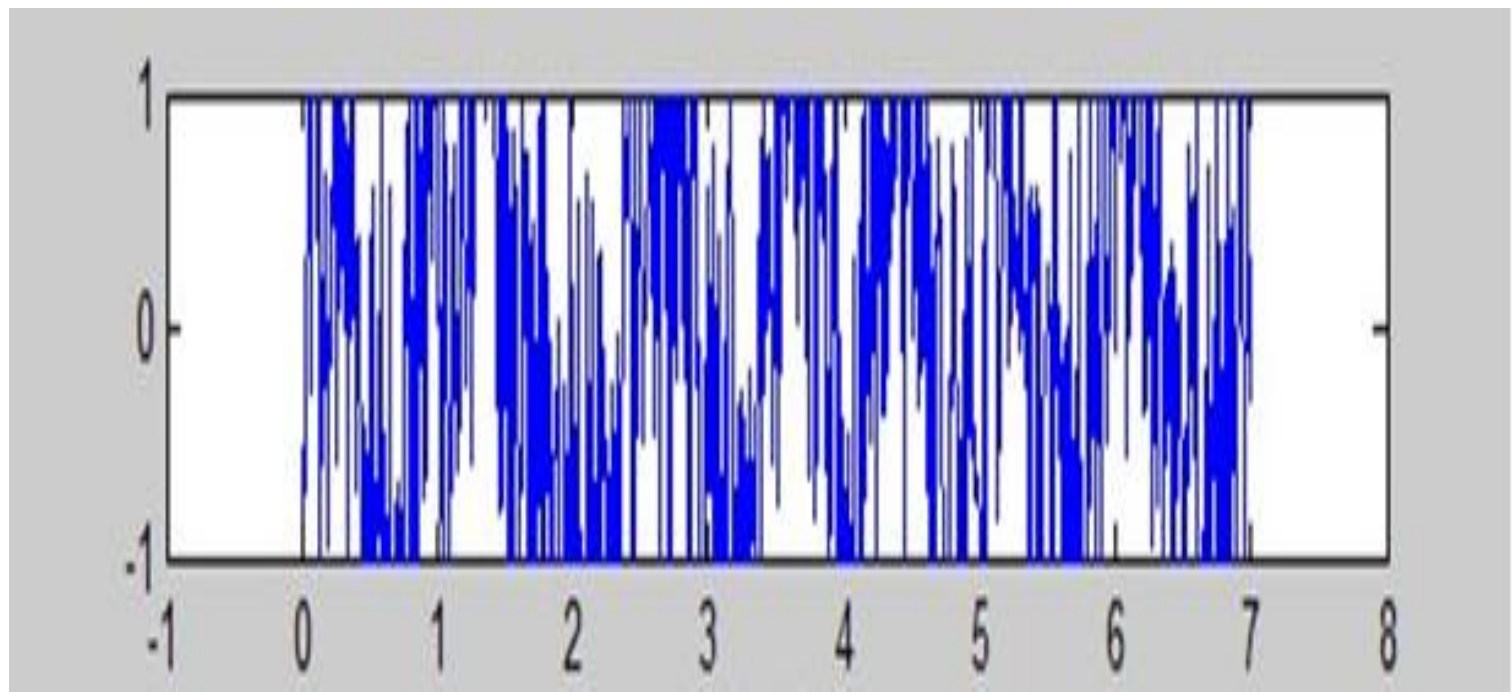

Figure 3. QPSK signal after the addition of noise 


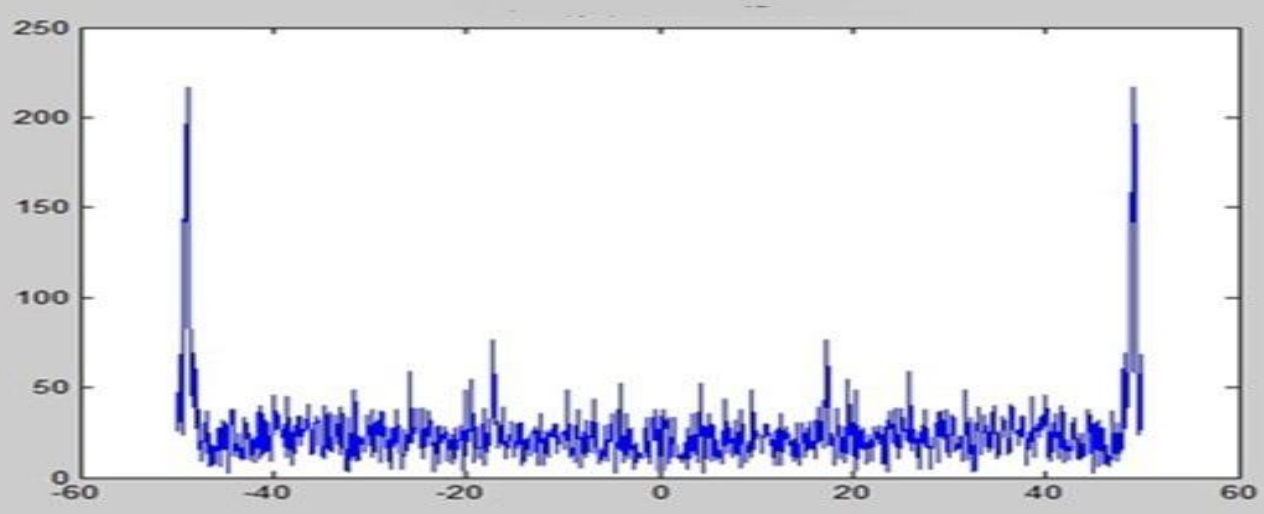

Figure 4. Spectrum of QPSK after the addition of noise

Conclusion: According to QPSK modulation process, there are fewer burrs on the spectrogram before the addition of noise. The spectrogram with the addition of noise has more burrs.

\section{REALIZATION OF QPSK SIGNAL DEMODULATION}

QPSK is subject to coherent demodulation here. That is, two signals respectively multiply by carrier and $\mathrm{I}(\mathrm{t})$ and $\mathrm{Q}(\mathrm{t})$ are obtained through low pass filter. After discriminated by sampling discriminator, they are synthesized into one signal. Therefore, the initial signal is restored.

I_demo=QPSK_rc_n $0 . * \cos \left(2 * \mathrm{pi}^{*} \mathrm{f} 1 * \mathrm{t} 1\right)$; \% Demodulation( coherent demodulation, multiply by carrier)

Q_demo=QPSK_rc_n $0 . * \sin (2 *$ pi*f $1 * t 1)$;

I_recover=conv (I_demo,xrc);

\%Low pass filter

Q_recover=conv $\left(\mathrm{Q} \_\right.$demo, $\left.x r c\right)$;
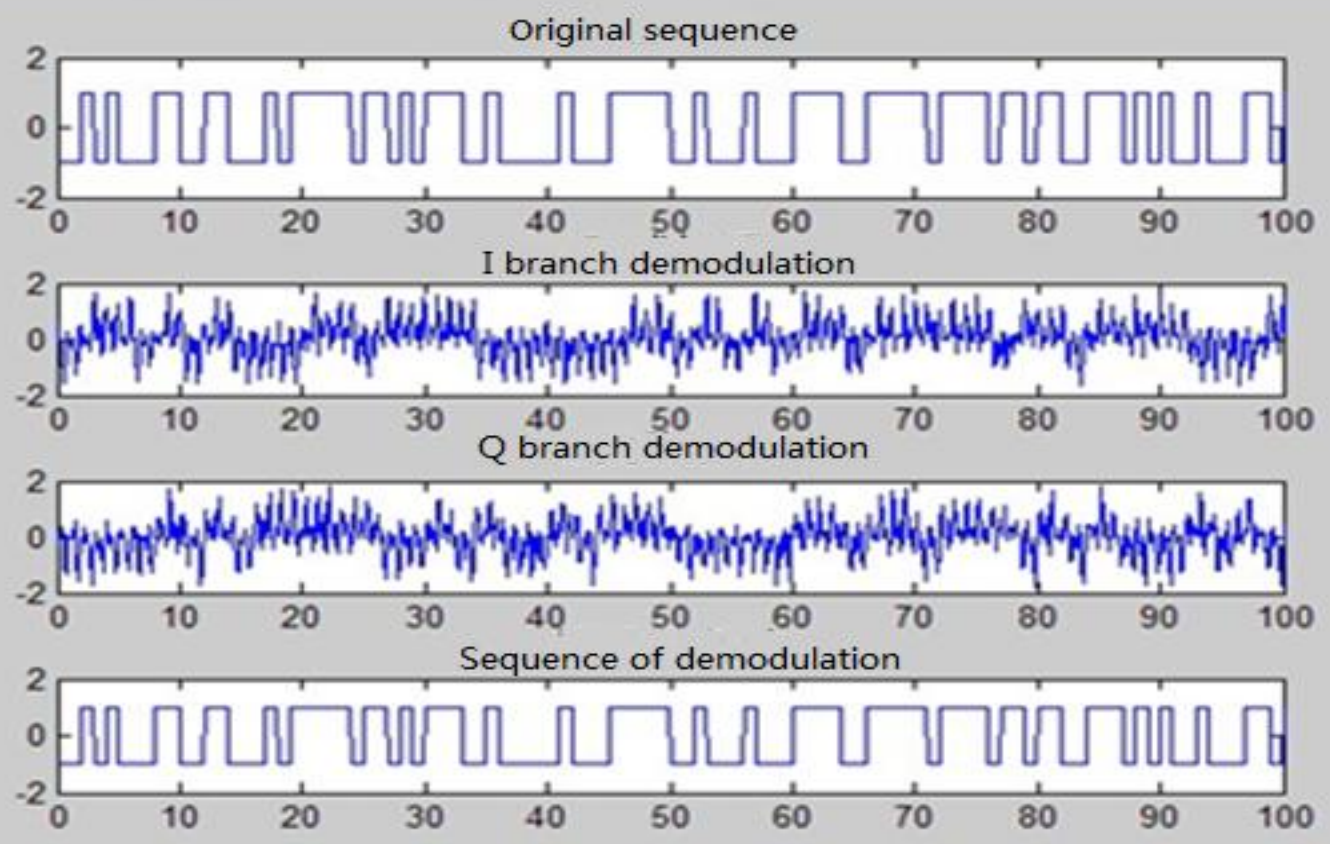

Figure 5. Comparison of QPSK demodulation signal and the original signal

According to the figure above, the demodulation of I and Q branches is the signal waveform with the addition of noise and without passing low pass filter. After correct demodulation, the final waveform does not have a high degree of distortion due to the introduction of evendistributed noise. The function involved is $\mathrm{n} 0=\operatorname{rand}(\operatorname{size}(\mathrm{t} 1))$. Some features can be obtained from
QPSK modulation and demodulation simulation Fig. 5. Coherent demodulation and modulation processes are basically reciprocal. Local carrier is introduced in both processes. It should be noted that carrier phases of two signals are orthotropic and the filters selected are low pass filters. 


\section{CONCLUSIONS}

Signals are processed in the design. They are subject to serial-parallel conversion and polarity conversion. Both signals respectively pass low pass filters and multiply by intrinsic signal. Therefore, two modulated signals are output. QPSK modulation signal is obtained through effective stacking in public time periods. Noise is often added in actual physical channel. Matlab function is used to simulate the introduction of random noise. The situation of noise spectrum before and after the addition of noise is observed on the simulation figure. In the demodulation process, QPSK modulated signal first multiplies by local carrier. It then passes low pass filter. Sampling discrimination is conducted then, the parallel signals are synthesized and output, and the original information is restored. Original information input by the transmitting terminal and the information restored after demodulation are compared. The figures show a very low degree of distortion.

\section{REFERENCES}

[1] Fan Changxin, Zhan Daoyong, Xu Bingxiang, Wu Chengke et al. Communication Principle (the 5th ediiton) [M]. Beijing: National Defence Industry Press, 2001.

[2] Wu Weijun. Key Technologies in Mobile Communication [M]. Beijing: Beijing University of Posts and Telecommunications Press 2000.

[3] Yan Bo, Cheng Hui, Liu Xuelin. High Speed Data Modulation and Demodulation Technology. Radio Communication Technology, Vol. 29. No.3. 2003.
[4] Written by (USA) Proakis, J.G. Translated by Zhang Lijun, Zhang Zongcheng, Zheng Baoyu et al. Digital Communication (the 4th edition). Beijing: Electronic Industry Press, 2003.

[5] Guo Xingyang, Xie Defang. Research on Correction Method for QPSK Digital Demodulation Phase Ambiguity [J]. Journal of Air Force Engineering University (Natural Science Edition), 2001,2(1):41-43.

[6] Zhang Jianwu. Mobile Communication. Xi' an University of Electronic Science and Technology Press, 2013.

[7] Wang Lining, Yue Guangxin, Zhan Fei. MATLAB and Communication Simulation [M]. Beijing: Posts and Telecom Press, 2000.

[8] Xu Mingyuan, Shao Yubin. Application of MATLAB Simulation in Communication and Electronic Engineering Simulation [M]. Shaanxi: Xi' an University of Electronic Science and Technology Press, 2005.

[9] Cao Zhigang, Qian Yasheng. Modern Communication Principle. Beijing: Tsinghua University Press, 1992.

[10] Zhou Jiongpan, Wu Weiling. Communication Principle. The 3rd Edition, Beijing: Beijing University of Posts and Telecommunications Press, 2006

[11] Huang Zheng, BPSK, QPSK and Their Demodulation [J]. Beijing: Posts and Telecom Press, 2000

[12] Gao Xueping, Guan Boran, Wang Haiyong. System Simulation Experiment of QPSK Modulation and Demodulation [J]. Journal of Hangzhou Electronic Science and Technology University, 2006.

[13] Wang Huakui et al. Mobile Communication Principle and Technology. Tsinghua University Press, January 2009.

[14] Wang Zhenglin et al. MATLAB/simulink and Control System Simulation. Electronic Industry Press. July 2008. 